\title{
Application of Skeletonization Algorithms for Myocardial Spect Quantification
}

\author{
Didier Scellier*, Jean-Yves Boire*, Cyril Thouly**, Jean Maublant** \\ * ERIM, rue Montalembert, BP 184, 63005 Clermont-Ferrand, cedex, France \\ ** Médecine Nucléaire, Centre Jean Perrin, BP 392, 63011 Clermont-Ferrand, France
}

\begin{abstract}
Defect quantification is an important topic in myocardial SPECT. It can be achieved properly only with an automatic and reproducible processing. We propose a new approach lying on the application of discrete geometry and of two aspects of mathematical morphology, namely, skeletonization and segmentation. The properties of the skeleton are essential for carrying out segmentation, which uses many sights of uncertain's theory (fuzzy logic). We present the basis of this application with an in-depth analysis of various methods of skeletonization.
\end{abstract}

KEY WORDS : Skeleton, fuzzy logic, segmentation, scintigraphy

\section{I- INTRODUCTION}

Myocardial single photon emission computed tomography (SPECT) consists in collecting a set of images by the rotation of a scintillation camera around a patient who has been previously injected with a radiotracer showing cardiac uptake. An algorithm of filtered backprojection allows the reconstruction of sections perpendicularly to the surface of the detector [1]. The distribution of the tracer in the left ventricular myocardium can be assessed from these images, which represent the anatomical structure in 3D (Fig.1). The object is usually presented with a color scale linked to the tissular activity. An abnormal area is characterized by a low level of activity. It is important for the diagnosis to be able to recognize the size, location and depth of the abnormal areas. Quantifying the activity implies to involve the entire myocardium which includes the normal areas as well as those with a low level of activity. One of the challenges of automatic analysis is to be able to detect these areas with a low activity, which could be mistaken with extra-cardiac tissue. Our program of quantification of the activity levels in the whole myocardium lies on a new approach combining fuzzy logic, discrete geometry and mathematical morphology.

- To obtain a 3D skeleton of the left ventricle (LV), the original images are first segmented with a fixed threshold at $50 \%$ of the maximum within the object. It is not necessary to use an optimal segmentation method because the value of this threshold has only a little influence on the resulting skeleton.

- The resulting skeleton is not necessarily connected because the patient can present a pathology in which connectivity can be lost. The defects of the skeleton are then filled according to the theoretical model of the 'truncated bullet' $[2,3]$.

- A new image made up, for each pixel, of its "probability of belonging to the LV" is calculated by comparing the points of the original image (fuzzy set) to the skeleton (no fuzzy set). The probability is estimated by taking into account the activity to the considered fuzzy point, the distance separating that point from the closest of the "no fussy points", and the value of each skeleton's point, called "shape function". 
This synthetic image permits to generate a deeper contrast between the normal and pathological walls, facilitating the segmentation of the whole LV. The volume is then sectorized and the quantitative value calculated for each sector (percentage of fixation relatively to the sector with highest value) allows the physicians to estimate the extent of the disease.

In this process, the skeleton plays a central role. It is used to adjust the theoretical model, and it is also a guide in fuzzy segmentation to increase the contrast. We will present several solutions and assess their capabilities.
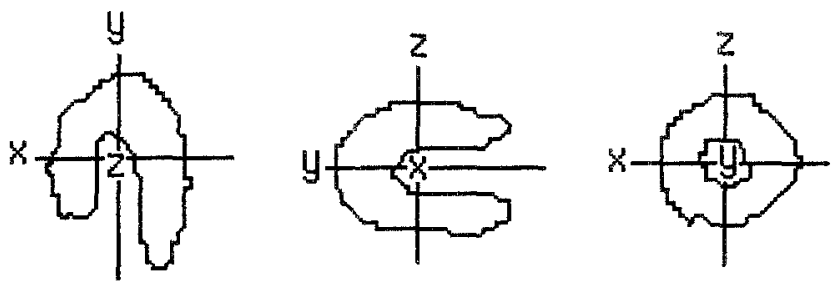

Fig.1 Outlines of a normal, reoriented, left ventricle on orthogonal sections.

\section{II-METHODS}

\section{1-DEFINITIONS}

An object is said " $n$-connect" if each point of this object has a neighbor in its nneighbor. Connectivities generally used are the 4-connectivity or 8-connectivity for the $2 \mathrm{D}$ space, the 6-connectivity and the 26-connectivity for 3D space [4].

A skeleton is a result of an object transformation into a threadlike representation [5]. A skeleton must have the following characteristics :

- it must be included and centered into the initial object,

- it must be thin,

- its transformation must be homotopic,

- its shape must be the same that the initial object,

- its transformation must be reversible.

The skeleton should permit a good recognition of the initial object. Its advantage lies in the diminution of the number of data.

\section{2- CLASSES OF METHODS}

In the discrete space, three classes are possible:

\section{The largest balls included in the object}

- fire propagation simulation,

- the object is exactly encoding,

- the transformation is reversible,

- but the obtained skeleton is generally no-connect. 


\section{Binary skeletons}

- object thinning. The topology is preserved when the strategy of "simple point suppression" is followed,

- algorithm is a translate of the wave propagation phenomenon,

- the transformation is homotopic,

- but the transformation is not directly reversible.

\section{Definition of medians lines}

- The skeleton is obtained from the distance map,

- association of the binary skeleton and largest balls approach,

- isotropy and reversibility are preserved simultaneously,

- but the obtained skeleton is not always thin.

\section{3- LEFT VENTRICULAR SKELETIZATION}

Defect quantification of myocardial SPECT supposes a good segmentation of all the organ. Areas with a high activity (normal areas) are easily segmented, but those with a low activity can be segmented only if the shape of the myocardium is known.

\section{Proposition 1 : using the skeleton's reversibility}

The first proposed quantification program [3] was only supported by the reversibility of the skeleton. The chosen algorithm of skeletonization belongs to the first class of method.

\section{Principle}

- original images are segmented by using an auto-adaptive threshold [6]. The binary object obtained with this segmentation represents the normal areas of the myocardium.

- The binary object is then skeletonized with the Lantuejoul's algorithm generalized to the 3D space [3] (Fig.2). This algorithm has been chosen for its fast execution and its possibility of reversibility because of the shape functions. It is this $3 \mathrm{D}$ skeleton which is used as a guide for the fitting to the ellipsoidal model. The skeleton is completed in accordance with the model. The shape function of the missing points is calculated by linear adjustment.

- The reversibility of the transformation permits to obtain a LV without defect in accordance with the supposed morphological aspect of the organ.

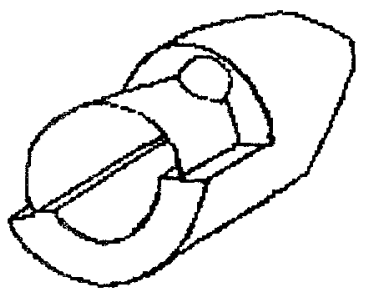

Fig.2 Skeletization by the Lantuejoul's algorithm of a 3D object with a spherical structurating element of size 1 . 


\section{Proposition 2 : using fuzzy logic}

The former program was using the advantages brought by the reversibility of the transformation without taking care of the other skeleton characteristics. The present approach associates the skeleton to other data such as the levels of activity in the image, and the algorithm of segmentation uses the basis aspects of fuzzy logic [7].

\section{Principle}

- The frames are segmented with a fixed threshold at $50 \%$ of the maximum pixel located in a region of interest. The level of this threshold has only a little effect on the skeleton appearance.

- The binary LV is then skeletonized. The choice of the method of skeletonization is essential. It must have simultaneously the three following properties:

* it must be thin, included and centered in the LV,

* it must have the same number of connected components that the LV,

* it must have the same shape that the initial object.

The skeleton is completed in accordance with the model. The added points are initialized with the value 1 , which allows to recognize the defect (strong diminution of activity).

Comment: The algorithm of fuzzy segmentation that we have developed has been tested only on a set of $2 \mathrm{D}$ images.

- The algorithm of fuzzy segmentation creates images in which the contrast is more pronounced. These images can be more easily segmented, the value of the threshold having much less influence on the result (Fig. 3).

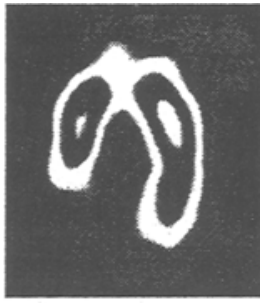

(a)

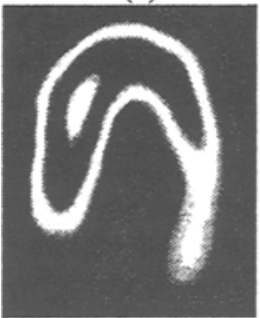

(c)

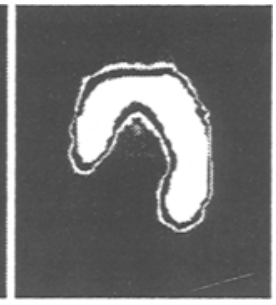

(b)

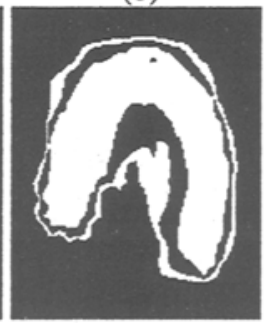

(d)
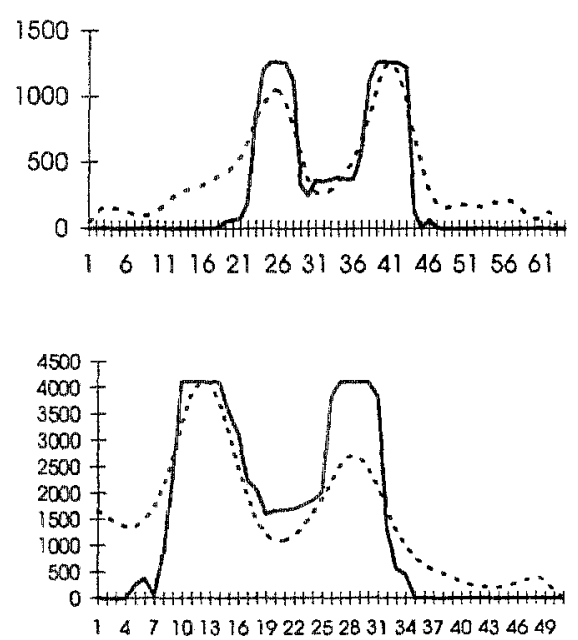

Fig.3 Comparison of contrast between original images (a),(c) and their respective transformation into images of affiliation probabilities (b),(d). Activity profiles of the original images are in dashed lines and probability images profiles in full lines. The area of decreased activity seen on the original image of view (c) disappears on its transformed image (d). 


\section{Theoretical principle of the fuzzy segmentation}

The algorithm is applied on a set of $2 \mathrm{D}$ images. The $3 \mathrm{D}$ object is obtained with the superposition of this set.

Input data : original images of the LV, and its skeleton.

Output data : a synthetic image composed with probability of affiliation to the myocardium.

Theoretical data :

1. points of the side image belonging to the myocardium: probability of affiliation $\mu=0$.

2. skeleton points belonging to the myocardium: probability of affiliation $\mu=1$

3. skeleton points and side points of the image composing the no-fuzzy set

4. all points which do not belong at the no fuzzy set are called fuzzy points and form the fuzzy set: probability of affiliation $\mu=[0,1]$.

For each fuzzy point, the probability of affiliation is computed from a distance covered in the 8 directions. For each direction, four data are extracted:

1. the activity level at the local fuzzy point $p$,

2. the activity level at the no fuzzy point encountered $i$,

3. the distance between the fuzzy point $p$ and the no fuzzy point $i, d(p / i)$,

4. the value of the shape function of the no fuzzy point encountered $f(i)$.

The probability of affiliation to the myocardium is calculated in two times :

1. For each direction, a first degree of affiliation $\mu \mathrm{i}(\mathrm{p})$ is calculated, according to the activity level:

$$
\mu^{\prime} \mathrm{i}(\mathrm{p})=|\operatorname{act}(\mathrm{p})-\operatorname{act}(\mathrm{i})| \beta
$$

$\beta$ : normalization coefficient.

2. The distance and the shape function are then used to calculate in each direction the degree of affiliation $\mu i(p)$ :

$$
\mu \dot{\mathrm{i}}(\mathrm{p})=\frac{\left(\mu^{\prime} \mathrm{i}(\mathrm{p})+\mathrm{k} \cdot \mathrm{f}(\mathrm{i})-\mathrm{p} \cdot \mathrm{d}(\mathrm{p} / \mathrm{i})\right)}{\sum_{\mathrm{i}=1}^{8}\left(\mu^{\prime} \mathrm{i}(\mathrm{p})+\mathrm{k} \cdot \mathrm{f}(\mathrm{i})-\mathrm{p} \cdot \mathrm{d}(\mathrm{p} / \mathrm{i})\right)}
$$

The probability for each point to belong to the myocardium is obtained by adding the $\mu \mathrm{i}(\mathrm{p})$ in which the directions are in touch with the skeleton. The result is an image of the probabilities of affiliation in which the contrast is increased and the apparent level of activity of the defects is attenuated (Fig.3-b). A threshold applied to that image provides the binary LV. 


\section{III-DISCUSSION}

\section{Proposition 1}

The Lantuejoul's skeleton (3D) is well suited to reconstitute the LV outlines. The skeleton does not have to be connected because the missing points are added by modelisation. The small branches are apparently not a problem because they are completely integrated to the object. The reversibility of the transformation allows to create a new object close to the supposed actual organ (fig.4). However the quantitative values obtained after sectorization were not always in agreement with the physician's visual analysis. This occurred mostly in the hypoactive areas characterized by an activity level too high to be considered as a defect, and too low to be considered as a normal area. After segmentation with an auto-adaptive threshold, the hypoactive areas are characterized by a wall thinning. Consequently some points in that zone are not well adjusted by the linear fitting of the shape function. After the reverse transformation, the obtained LV always get this thin wall. We can conclude that the program is not efficient if the skeleton alone is considered. It is necessary to introduce intrinsic parameters to the object such as activity levels. This is why a second approach has been studied, combining discrete geometry, mathematical morphology and fuzzy logic.

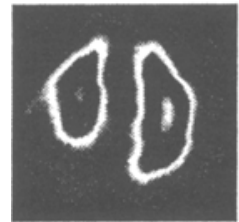

(a)

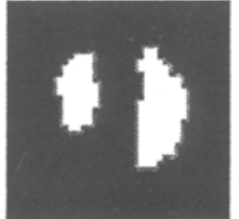

(b)

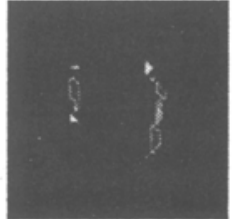

(c)

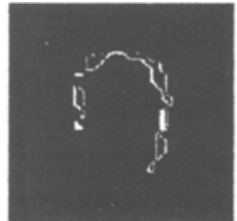

(d)

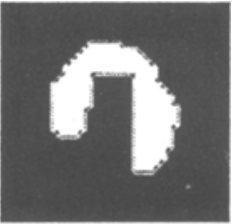

(e)

Fig.4 : Aspects of the different steps of the first quantification method.

(a) original image of a $\mathrm{LV}$ slice

(b) segmentation of normal areas with an auto-adaptive threshold

(c) skeleton of the binary LV obtained with the Lantuejoul's algorithm adapted in $3 \mathrm{D}$

(d) skeleton filling in accordance with the ellipsoidal model

(e) coming back to the initial object by the reversibility of the Lantuejoul's algorithm.

\section{Proposition 2}

The fuzzy segmentation algorithm can only be applied to a connected skeleton. In effect, the probability of affiliation is calculated by measuring the discrete distance separating the considered point and the skeleton point on the nearest side. A disconnection in the skeleton necessarily generates errors (Fig.5). The 2D or 3D Lantuejoul algorithm does not permit to obtain connexe skeletons. To use this method, the areas of disconnection must be filled in. This can be achieved through modelisation. When one point is added, it is positioned at 1 , a value indicating a defect. By connecting the skeleton with modelisation, the points situated in normal zones could be considered as defects. An "ebarbulage" (cutting away the small branches) allows to eliminate the rebellious points (Fig.6). But in many cases, the "ebarbulage" creates new disconnection's. The first class of method is so not directly applicable. 


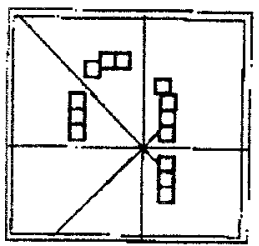

Fig.5 Ray tracing in the case of a skeleton disconnection.

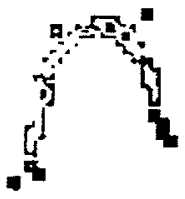

(a)

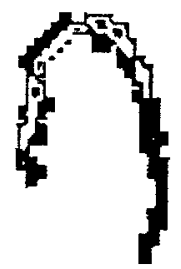

(b)

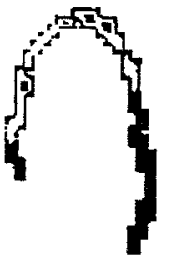

(c)

Fig.6 The patient skeleton (a) is filled in accordance with the ellipsoidal model (b) and then "ebarbulated" (c)

The third class of method consists in using the distance mapping. The purposed algorithm generally calculates the skeleton in two steps. The first one consists in finding an initial sub-entity of the skeleton, like the local maxima on the distance mapping. As the obtained sub-entity is unconnected, a second step tries to get it connected. In $2 \mathrm{D}$, the skeletonization of the LV slices gives satisfying results (Fig.7). The skeleton is connected, and the distance mapping permits to locate the wall thickness.

The second class of method consists in thinning the object but by keeping its initial topology. Generally, the skeletonization algorithm by thinning creates binary skeletons. The advantage of these algorithm is to be able to choose the kind of connectivity. The 2D skeletonization of the LV slices permits to obtain a perfectly 4-connexed skeleton (Fig.8). Although the skeleton is binary, the thickness of the wall can be obtained by superposition of its points with the distance mapping of the object.

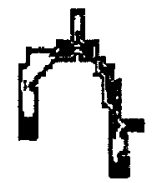

Fig.7 2D skeleton obtained with the distance mapping<smiles>C=CCc1ccccc1CCCC</smiles>

Fig.8 2D skeleton obtained with the homotopic thinning method 
The fuzzy segmentation algorithm is applied to a set of $2 \mathrm{D}$ images. Is it necessary to apply skeletonization to another dimension ? The modelisation step must be applied on 3D skeleton. The presence of a little branch ("barbule") on the superior part of the skeleton generates a morphologic incompatibility between the skeleton and its tronquated bullet model (Fig.9). This little branch is not really a barbule because it is the logical result of a 2D LV skeletonization. Fuzzy segmentation algorithm assumes that the skeleton is morphological like the initial object. All skeletonization methods applied in 2D (Fig.10) do not permit to consider the object in its supposed shape. On the three methods used, the first is already apply in 3D (Fig.11). The obtained skeleton is more unconnected. The third method class is very difficult to adapt to $3 \mathrm{D}$ because of the important number of configurations to test at the reconnection step of the local maximum.
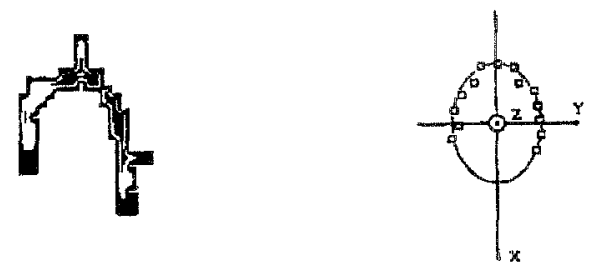

Fig.9 2D slice of LV skeleton and its 3D theoretical model.
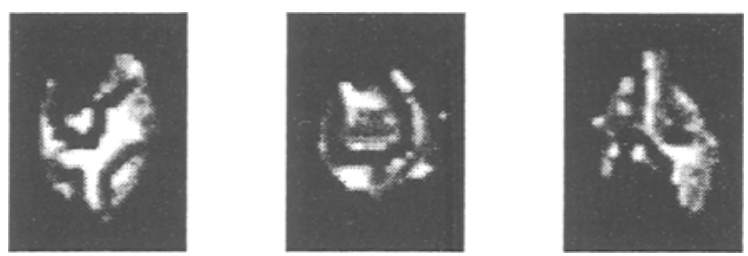

Fig.10 3D visualization obtained with $2 \mathrm{D}$ skeleton superposition.
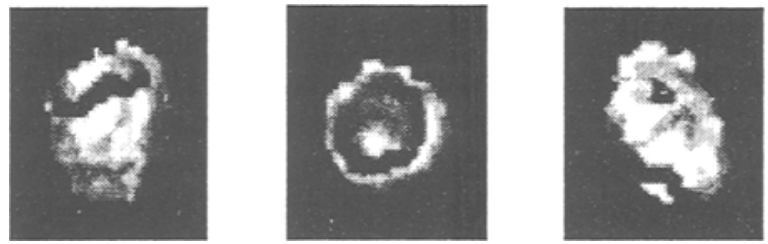

Fig.11 3D visualization obtained with 2D skeleton superposition.

In the second class of methods, [9] gives a precise definition of $\mathrm{p}$-simple-points for 3D space. These points can be deleted without modifying the object topology. The algorithm is quick, reliable, and the object topology is preserved. The skeleton is n- 
connected and $\mathrm{n}$ can be chosen by the user. In our case, a 6-connected skeleton should solve our problems. A 3D skeletonization algorithm is presently being studied [10].

All methods presents in this paper are applied in the discrete space. If continuous or semi-continuous space is considered, the number of methods proposed is more important. [8], [12], [13].

But the continuous space is not well adapted for our application because of the space resolution of the original images is too many small $(25 * 25 * 32$ pixels maximum).

\section{IV-CONCLUSION}

Presently, 3D Lantuejoul skeletonization is utilized in our fuzzy segmentation algorithm. The obtained skeleton is unconnected and has a lot of "barbules". It cannot be used in this state. In a first step, the skeleton is ebarbuled. It is then filled in accordance with the theoretical model. The added points are set to 1 . Eventually the skeleton is again ebarbulated (Fig.6). Our fuzzy segmentation program better fill defects than the former method. Qualitative results estimated by a specialized physician show that hypoactive areas are better detected, and myocardial defects better filled. But for a lot of patients filling problems subsist, especially in relation with the choice of method of skeletonization. We have shown in our analysis that the Lantuejoul algorithm, as well as the first class of methods, are not well suited for our application. We are searching for an algorithm which associates the following characteristics :

- homotopic transformation,

- shape identical to the initial object,

- distance to the border should be available.

It is very difficult to implement all these criteria in the same algorithm. Among all the methods described, only two should be able to bring a solution to our problems $[8],[9],[10],[12],[13]$. These methods have the advantage to have been developed for 3D's objects.

Skeletonization is an essential part for the processing of our myocardial SPECT images. It is necessary for the modelisation and it provides the basis for the fuzzy segmentation algorithm. We envision other applications of this method for automatic centering of the heart image, and also to develop a quantitative analysis a wall motion based on the "center-line" method [11].

\section{REFERENCES}

[1] Helias J, Itti R, Pezard Ph, Coeur et isotopes. Médicorama, Numéro spécial. 1988.

[2] Cauvin JC, Boire JY, Maublant J, et al. Automatic detection of the left ventricular myocardium long axis and center in thallium-201 single photon emission computed tomography. Eur. J. Nucl. Med. Vol. 19, N 12 , December 1992: pp 1032-1037.

[3] Cauvin JC, Boire JY, Maublant J, et al. Automatic quantification of myocardial defects by morphological methods in SPECT. Proceedings of the 12th annual international conference of the IEEE Engineering in Medicine an Biology Society. Philadelphia, PA, $1990: 415-416$.

[4] Rosenfeld A, Three-dimensional digital topology. Tech. Rpt. 936, Computer Science Center, University of Maryland, College Park, MD 20742, September. 
[5] Chassery JM, Montanvert A, Géométrie discrète en analyse d'images. Traité des nouvelles technologies serie - Images, Hermes, 1991.

[6] Boire JY, Cauvin JC, Maublant J, et al. Segmentation methods for automatic kidneys volume quantification in SPECT. Proceedings of the 12th annual international conference of the IEEE Engineering in Medicine an Biology Society. Philadelphia, PA, 1990:421-422.

[7] Faurous P, Fillard JP, Artus A, A new approach to fuzzy partition for image segmentation. International Protocol Conference of the IEEE Enginering in Medecine and Biology Society, Vol.14, Part 5 to 7, pp 1922-1923, Paris, oct-nov 1992.

[8] Fernandez-Vidal S, Malandain G, Squelettes euclidiens d'objets discrets nDimensionnels

Rapport de Recherche INRIA N²771, Janvier 1996.

[9] Bertrand G, Simple points, topological numbers and geodesic neighborhoods in cubic grids

Pattern Recognition letters 15, pp 1003-1011, 1994.

[10] Bertrand G, P-simple points : a solution for parallel thinning. Actes du 5ième Colloque DGCI, pp 233-242, sept 1995

[11] Sheehan F.H, Bolson E.L, Dodge H.T, Detlef G, Schofer J, Woo H, Advantages and applications of the center line method for characterizing regional ventricular function. Circulation 74, N², 293-305, 1986.

[12] D. Attali and A. Montanvert : Semi-continuous skeletons of $2 \mathrm{D}$ and $3 \mathrm{D}$ shapes. In C. Arcelli et al editors, Aspects of Visual form Processing, page 32-41. World Scientific, Singapore 1994

[13] D. Attali and A. Montanvert : Computing and symplifying 2D and 3D semi-continuous skeletons. CVGIP : Image understanding, 1995 